\title{
Underlying Assets Distribution in Derivatives: The BRIC Case
}

\section{José Antonio Núñez*, Mario I. Contreras-Valdez, Alfredo Ramírez-García, Eduardo Sánchez-Ruenes}

Tecnológico de Monterrey, EGADE Business School, Mexico City, Mexico

Email: `janm@itesm.mx

How to cite this paper: Núñez, J.A., Contreras-Valdez, M.I., Ramírez-García, A. and Sánchez-Ruenes, E. (2018) Underlying Assets Distribution in Derivatives: The BRIC Case. Theoretical Economics Letters, 8, 502-513. https://doi.org/10.4236/tel.2018.83035

Received: December 7, 2017

Accepted: February 11, 2018

Published: February 14, 2018

Copyright (c) 2018 by authors and Scientific Research Publishing Inc. This work is licensed under the Creative Commons Attribution International License (CC BY 4.0).

http://creativecommons.org/licenses/by/4.0/

\section{cC) (i) Open Access}

\begin{abstract}
This paper addresses one of the main issues regarding numerical derivatives valuation, particularly the search for an alternative to the normality assumption of underlying asset returns, to obtain the price by using numerical techniques. There might be difficulties in making normality assumptions, which could produce over-valuated or sub-valuated prices of derivatives. Under this consideration, the Generalized Hyperbolic family has been proven to be a proper selection to model heavy tailed distribution behavior. The Normal Inverse Gaussian (NIG) distribution is a member flexible enough to model financial returns. NIG distribution can be used to model distribution returns under different states of nature. The indexes of the Brazil, Russia, India and China (BRIC) economies were studied at different time-periods using return data series from 2002 to 2005, 2006 to 2010 and 2011 to 2015, in such a manner to demonstrate with statistical criteria that NIG fits the empirical distribution in the three periods; even throughout economic downturn. This result may be used as an improvement in derivatives valuation with indexes as underlying assets.
\end{abstract}

\section{Keywords}

Derivatives, Normal Inverse Gaussian, Valuation, Returns, BRIC

\section{Introduction}

One of the main concerns regarding financial analysis, derives from an inadequate study of the returns of several financial variables; since it is imperative to model the dynamics of these returns. With the continuous development of mathematical tools as well as computational capacity, different approaches have attempted to improve model adjustment by combining numerical and analytical 
frameworks. In particular, the stochastic processes modelling returns have been the core of the researching agenda development since the thesis was proposed by Bachélier in 1900 [1]. Premise, in which normality is assumed, has been proven to be erroneous by application of diverse statistical tests. This issue is transferred to another branch: the derivatives valuation. In numerous cases, the valuation of different derivatives is tested by Monte Carlo simulation as they do not have a close valuation formula; i.e. there is not an exact equation, as in the case of European Options with the well-known Black, Scholes and Merton model. For Monte Carlo's application, it is necessary to investigate, for example, stochastic returns dynamics and their respective simulation algorithm.

In this context, the importance of derivatives valuation has become a central issue to consider. According to data from World Bank, the estimated global GDP in 2016 was placed at 75.543 billion dollars. In addition, the BIS published their semiannual derivative statistics, in which the approximate notional amount of outstanding OTC derivatives contracts equaled $\$ 542$ trillion dollars at the end of June 2017 [2]. Noting this disparity, the relevance of studies around derivatives valuation in modern world becomes quite evident.

In this regard, multiple studies researched for better approximations to empirical returns density and have demonstrated that the members of the Generalized Hyperbolic family have a better fit to observations [3] [4]. One of the main properties regarding this distributions set is the presence of heavier tails than those of Normal distribution; which is an important property of returns' behavior [5]. Another property is the flexibility of the functions, since these distributions are determined by five parameters. The adjustment has a better opportunity to reflect the nature of the empirical data; property that according to Cont, is necessary to approach this kind of distributions [5].

Although the Normal Inverse Gaussian (NIG) distribution has verified to be an excellent selection for stock returns distribution, it may also be used inclusively for indexes' returns of financial markets [6] [7].

In particular, this paper focuses on the probability distribution model of the main indexes of the BRIC economies in different periods of financial conditions (the case of South Africa is excluded because it was not until 2010, that it became part of this group).

By dividing this study into three time intervals, the period from 2002 to 2015, we reviewed the capability of NIG to fit distribution returns even in extreme periods, such as the downturn of the financial crisis. By doing so, it may be possible to use Monte Carlo simulations with the proper distribution and parameters in order to obtain a better price of derivatives which relies on index as underlying asset.

The organization of the paper follows the next sequence: in Section 2, we discuss the Generalized Hyperbolic and Normal Inverse Gaussian distributions. The methodology applied to the data is presented in Section 3; then, Section 4 shows the results obtained. Finally, the conclusions are stated in Section 5. 


\section{Literature Review}

The Generalized Hyperbolic family was firstly proposed by Barndorff-Nielsen in 1977 [8]. This class of distributions is defined by five parameters; by fixing the parameter $\lambda=-1 / 2$, the NIG distribution is obtained. He exposed the capability of the NIG distribution to model heavier tails than those of the Normal distribution, a fact which is commonly found in returns data series. These characteristics became of interest due to the consequences in risk management applications and other branches of finance. Particularly Eberlein \& Keller used the DAX index in a three-year period from 1989 to 1992 to perform statistical test to compare the fit of empirical data with adjusted Normal and Hyperbolic distributions [3]. Their results concluded that, for the studied period, the hyperbolic distribution is a better option to model returns.

However, later studies Barndorff-Nielsen used NIG distributions as it is better to model heavy tailed observations [9]. Under this assumption he used statistical tests as well, in order to compare how Hyperbolic and NIG distributions fit to empirical data. Finally, he concluded that NIG performs better as a model for the same data used by Eberlein and Keller [9]. Later, Rydberg proved (using data from Denmark, Germany and United States' stock markets) that the NIG distribution is a better function to model the returns [10].

Trejo, Núñez and Lorenzo developed a study around the usage of NIG to model the stock return distribution in the Mexican market, as well as the IPC and S \& P500-indexes from Mexico and United States respectively [11]. By studying the Mexican market, they were able to show that NIG distribution has a better fit for stocks and index return distribution than a Normal one; so tools like the Brownian Process are not the best to simulate Mexican financial series.

Recent studies concerning the Generalized Hyperbolic family of returns in prices of commodities such as gold and petroleum have been done. Mota and Mata use historical prices from Brent, WTI and Mexican mix, to adjust the parameters of a HG distribution [12]. For this purpose, they took two-time period intervals to determine if those fit can be used in different states of nature; using the period from 2010 to 2013 a higher price, and from 2014 to 2015 a period in which the international price plummeted. Mota and Mata's results demonstrated that these types of distributions are able to better fit empirical returns of such commodities.

Relative to gold return, the studies have been conducted around the possibility to model distribution in order to obtain a better Value at Risk (VaR) measure [13] [14]. Using the time interval from 1991 to 2017, the studies use different classes of the Generalized Hyperbolic family, in order to compare them within risk management applications and techniques.

\section{Methodology}

For the purpose of this study, we used the daily data collected from Bloomberg of the BRIC indexes: IVOB, NIFTY50, SHCOMP and RTSI. Through the selec- 
tion of data series, we intended to represent three periods of time, in a manner to demonstrate that NIG distribution could fit indexes return data series in all nature states; the periods were named as pre-crisis (2002-2006), crisis (2007-2010) and post-crisis period (2011-2015). These representing periods were carefully chosen in order to shelter against other impacts not considered as part of the study. In particular, the crisis of 2001 emerged after the terrorist attack of the Twin Towers, and 2016 as the year in which capital flows returned to developed countries-mainly the continental United States-with the imminent interest rate normalization period [15] [16]. The nature of these shocks is distinct, but this work focuses only on the crisis included in the period from 2007 to 2010, thus naming the studied periods.

From each index series we calculated the logarithmic return as daily data could be used as an approximation to a continuous series; the equation states as follows:

$$
r_{i}=\ln P_{i}-\ln P_{i-\Delta t}, \quad \forall i=\{1, \cdots, n\}
$$

where:

$r_{i}$ is the return of the index on the day.

$P_{i}$ is the closing level of the index at day $i$.

$P_{i-1}$ is the closing level of the index at day $i-1$.

\subsection{Descriptive Statistics}

Skewness and kurtosis were calculated for each index series in order to validate distributions which have higher values of skewness, whether positive or negative; so we can expect that the empirical data does not correspond to a Normal distribution.

The statistics of these series are presented in the following Table 1.

Table 1. Descriptive statistics.

\begin{tabular}{cccccc}
\hline Time Period & Index & Mean & Variance & Skewness & Kurtosis \\
\hline \multirow{2}{*}{$2002-2005$} & IBOV & 0.0009 & 0.0003 & -0.2645 & 0.6944 \\
& NIFTY50 & 0.0011 & 0.0002 & -1.0271 & 9.0648 \\
& SHCOMP & 0.0004 & 0.0002 & 0.6407 & 4.6071 \\
& RTSI & 0.0015 & 0.0003 & -0.6751 & 4.1838 \\
& IBOV & 0.0006 & 0.0004 & -0.0031 & 6.1839 \\
& NIFTY50 & 0.0004 & 0.0004 & 0.0780 & 7.1262 \\
& SHCOMP & 0.0000 & 0.0004 & -0.2815 & 2.1921 \\
& RTSI & -0.0001 & 0.0007 & -0.3191 & 10.7541 \\
& IBOV & -0.0004 & 0.0002 & -0.0468 & 1.5607 \\
& NIFTY50 & 0.0002 & 0.0001 & -0.1697 & 1.5807 \\
& SHCOMP & 0.0002 & 0.0002 & -0.8859 & 6.2777 \\
& RTSI & -0.0007 & 0.0004 & -0.3010 & 6.7127 \\
\hline
\end{tabular}

${ }^{\star}$ High Kurtosis values appeared in every data series distribution. 
By analyzing the excess of kurtosis, a different behavior is notable from the Normal distribution, so the presence of heavy tails is expected.

\subsection{Normality Test}

Using the Anderson-Darling and Shapiro-Francia normality test, it is possible to reject the null hypothesis of normality. In this case the proposed NIG distribution becomes a candidate to fit the empirical data. Both normality tests are assumed as follows:

$\mathrm{H}_{0}$ : Sample resulting from a normal distribution is confirmed.

$\mathrm{H}_{\mathrm{a}}$ : Sample which does not come from a normal distribution, $\mathrm{H}_{0}$ is discarded.

For the acceptance of the null hypothesis, the p-value of each of the data series was obtained using both tests (Anderson-Darling and Shaphiro-Francia), with a level of significance of 0.05 , so that, if $p$-value $\geq 0.05$, the null hypothesis is accepted, otherwise it is rejected and the alternative hypothesis is accepted.

\subsection{Shapiro-Francia Test}

The normality test developed by Shapiro and Francia as an approximate and simplified version of the Shapiro Wilk test to prove the normality of a larger series of data [17]. The test parameter is obtained by calculating the slope of the regression line by simple least squares, i.e.,

$$
W=\left(\sum_{i=1}^{n} b_{i} y_{i}\right)^{2} / \sum_{i=1}^{n}\left(y_{i}-\bar{y}\right)^{2} .
$$

\subsection{Anderson Darling Test}

The Anderson-Darling criteria are used to test the hypothesis that a series of data comes from a population that adheres to a continuous Cumulative Distribution Function (CDF) [18]. The test is performed as follows:

$$
W_{n}^{2}=-n-\frac{1}{n} \sum_{j=1}^{n}(2 j-1)\left[\ln \left(u_{j}\right)+\ln \left(1-u_{n-j+1}\right)\right] .
$$

\subsection{Normal Inverse Gaussian Distribution}

As mentioned before, the NIG distribution has been proven in multiples studies to fit the financial series. This kind of distribution is defined by Barndorff-Nielsen as follows:

$$
g(x ; \alpha, \beta, \mu, \delta)=a(\alpha, \beta, \mu, \delta) q\left(\frac{x-\mu}{\delta}\right)^{-1} K_{1}\left\{\delta \alpha q\left(\frac{x-\mu}{\delta}\right)\right\} \exp (\beta x)
$$

where

$$
a(\alpha, \beta, \mu, \delta)=\pi^{-1} \alpha \exp \left(\delta \sqrt{\alpha^{2}-\beta^{2}}\right)-\beta \mu .
$$

And

$$
q(x)=\sqrt{1+x^{2}}
$$

where $K$ is the modified Bessel function of third order and index 1. Also, 
$\alpha, \beta, \mu$ and $\delta$, are parameters, satisfying $0 \leq|\beta| \leq \alpha, \mu \in \mathbb{R}$ and $0<\delta$.

The parameters $\alpha$ and $\beta$ determines the shape, and $\mu$ and $\delta$ scale the distribution. Parameter $\alpha$, which takes nonnegative values, denotes the flatness of the density function, i.e. a high value of $\alpha$ means a greater concentration of the probability around $\mu$. The parameter $\beta$ defines a kind of skewness of the distribution. When $\beta=0$, the NIG distribution is symmetric around the mean. A negative value represents a heavier left tail. The parameter $\delta$ describes the scale of the distribution and the parameter $\mu$ is responsible for the shift of the distribution density [6].

\subsection{Goodness of Fit Tests}

By simulating a vector with the obtained parameters, we test the similarity of both distributions with Kolmogrov-Smirnov and Anderson-Darling criteria in which the $p$-values corresponds with the acceptation zone of the null hypothesis. So according to the statistical tests, it is possible to say that NIG distribution is capable of modeling the returns even during a period of economic crisis.

\section{Results}

Through the results obtained by calculating the descriptive statistics of the series, we concluded that all series have heavy tails and high skewness values, which indicated that the series could be fitted with a member of the Hyperbolic Generalized Family; as the NIG distribution. Before we proceeded to estimate the NIG parameters, we applied the Normality Test (Anderson-Darling, Shapiro-Francia), to confirm that the series were not Normal.

Applying the Normality Test to the data it was possible to reject the null hypothesis of normality in every case. This result is consistent with the stylized features of financial series where higher values of kurtosis do not correspond to the Normal distribution (Table 2).

Table 2. Normality test ( $p$-value).

\begin{tabular}{cccc}
\hline Time Period & Index & Anderson-Darling & Shapiro-Francia \\
\hline \multirow{2}{*}{$2002-2005$} & IBOV & $5.783 \mathrm{e}-06$ & $1.55 \mathrm{e}-05$ \\
& NIFTY50 & $<2.2 \mathrm{e}-16$ & $<2.2 \mathrm{e}-16$ \\
SHCOMP & $<2.2 \mathrm{e}-16$ & $<2.2 \mathrm{e}-16$ \\
& RTSI & $<2.2 \mathrm{e}-16$ & $<2.2 \mathrm{e}-16$ \\
$2006-2010$ & IBOV & $<2.2 \mathrm{e}-16$ & $<2.2 \mathrm{e}-16$ \\
& NIFTY50 & $<2.2 \mathrm{e}-16$ & $<2.2 \mathrm{e}-16$ \\
& SHCOMP & $<2.2 \mathrm{e}-16$ & $1.62 \mathrm{e}-14$ \\
& RTSI & $<2.2 \mathrm{e}-16$ & $<2.2 \mathrm{e}-16$ \\
& IBOV & $4.85 \mathrm{e}-09$ & $3.502 \mathrm{e}-09$ \\
& NIFTY50 & $8.357 \mathrm{e}-09$ & $5.173 \mathrm{e}-09$ \\
& SHCOMP & $<2.2 \mathrm{e}-16$ & $<2.2 \mathrm{e}-16$ \\
& RTSI & $<2.2 \mathrm{e}-16$ & $<2.2 \mathrm{e}-16$ \\
\hline
\end{tabular}

Both Tests assume a significance level of 0.05 , that means that if $p$-value $\geq 0.05$ null hypothesis is accepted otherwise is rejected and the alternative hypothesis is confirmed. Own elaboration, data processed in $\mathrm{R}$ Software. 
Having proven the no-normality of indexes return distribution, and the excess kurtosis obtained from the descriptive statistics of the series, the NIG could be used to model the empirical data to obtain a distribution that better describes the empirical data series.

For the estimation of the NIG parameters we applied Maximum Likelihood Estimation (MLE). Although other methods could have been used, the selected algorithm solves the maximization problem by numerical methods. The parameters are shown in Table 3 below. Parameters were estimated using R Software.

With the estimated NIG parameters, a series with a particular NIG distribution was simulated in order to make a statistical analysis using Log-likelihood Test to compare the similarity of the empirical data series with the simulated data series. To complete this task, Kolmogorov-Smirnov and Anderson-Darling tests were used.

The results do not refuse the null hypothesis - the statistical similarity of distributions-in all cases except for the SHCOMP index in the pre-crisis period under the Kolmogorov-Smirnov test. However, the Anderson-Darling, considered a more precise test, confirms similarity in every case. Therefore, according to statistical criteria, the NIG distribution can fit the indexes return distribution in the three states of nature; defined as pre-crisis, crisis and post-crisis.

Quantitative results of Likelihood (Table 4) between NIG simulation distribution and the empirical data distribution obtained through the analysis could be observed graphically. Qualitative comparison of distributions considered Normal Distribution (red), empirical data distribution (blue) and simulated NIG distribution (green), as well as the QQ Plot is presented in the graphics bellow (Figures 1-3).

Table 3. NIG parameters.

\begin{tabular}{ccccccc}
\hline $\mathbf{p}$ & Index & $\boldsymbol{\alpha}$ & $\boldsymbol{\beta}$ & $\boldsymbol{\delta}$ & $\boldsymbol{\mu}$ & $\boldsymbol{n}$ \\
\hline \multirow{2}{*}{$2002-2005$} & IBOV & 115.3831 & -20.6768 & 0.0325 & 0.0068 & 1043 \\
& NIFTY50 & 79.7261 & 0.19 .269330 & 0.0136 & 0.0044 & 1258 \\
& SHCOMP & 56.8119 & 5.7093 & 0.0097 & 0.0006 & 1303 \\
& RTSI & 47.6604 & -7.6627 & 0.0146 & 0.0039 & 1303 \\
& IBOV & 31.8151 & -3.1540 & 0.0131 & 0.0019 & 1304 \\
& NIFTY50 & 35.9781 & -2.9873 & 0.0145 & 0.0016 & 989 \\
& SHCOMP & 40.5741 & -9.4047 & 0.0175 & 0.0042 & 1044 \\
& RTSI & 19.5138 & -2.9695 & 0.0139 & 0.0021 & 1044 \\
& IBOV & 99.0900 & 5.1516 & 0.0199 & -0.0014 & 1303 \\
& NIFTY50 & 124.5000 & -3.9450 & 0.0141 & 0.0007 & 1238 \\
& SHCOMP & 37.4145 & -1.1891 & 0.0081 & 0.0004 & 1303 \\
& RTSI & 44.6705 & -0.9362 & 0.0158 & -0.0003 & 1303 \\
\hline
\end{tabular}

NIG Parameters obtained for each series at different period times. Own elaboration, data processed in R Software. 


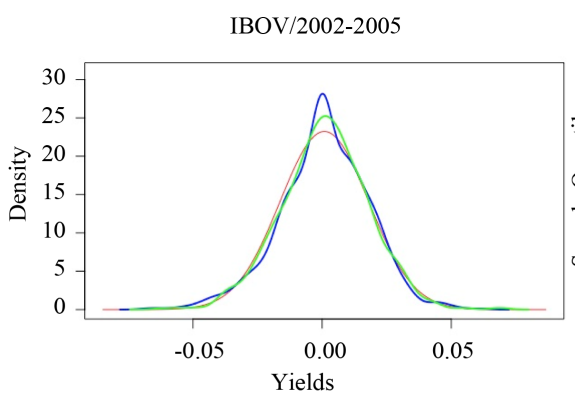

NIFTY50/2002-2005

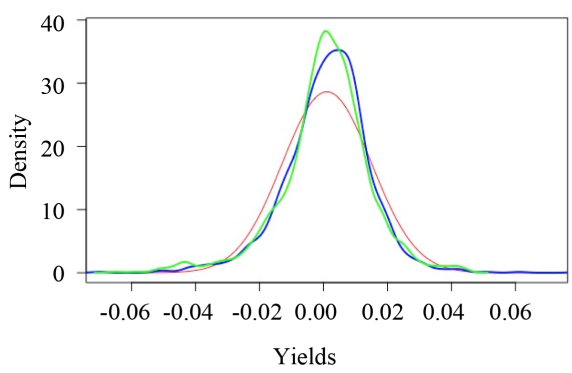

RTSI/2002-2005

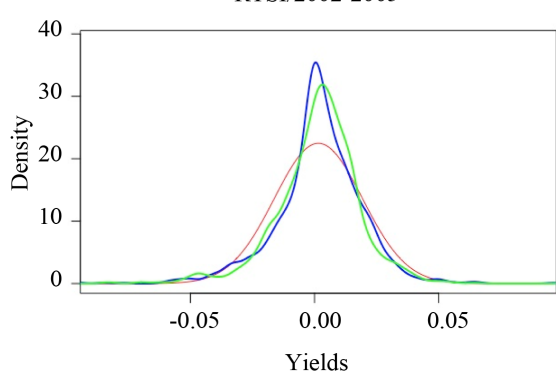

SHCOMP/2002-2005

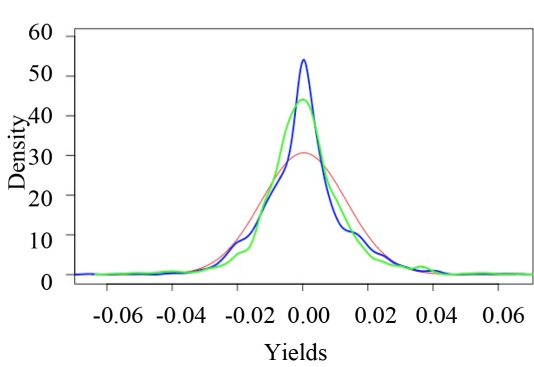

Q-Q Plot of IBOV/2002-2005

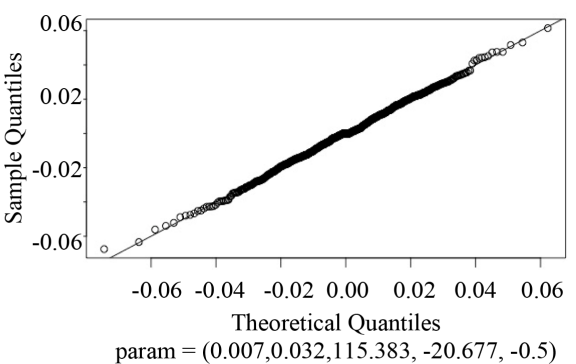

Q-Q Plot of NIFTY50/2002-2005

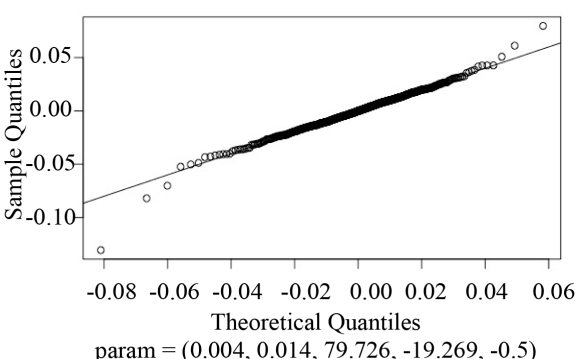

Q-Q Plot of RTSI/2002-2005

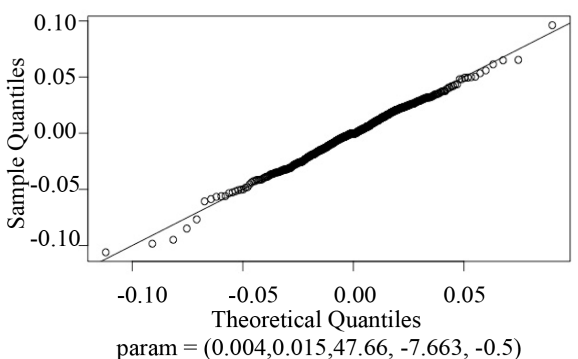

Q-Q Plot of SHCOMP/2002-2005

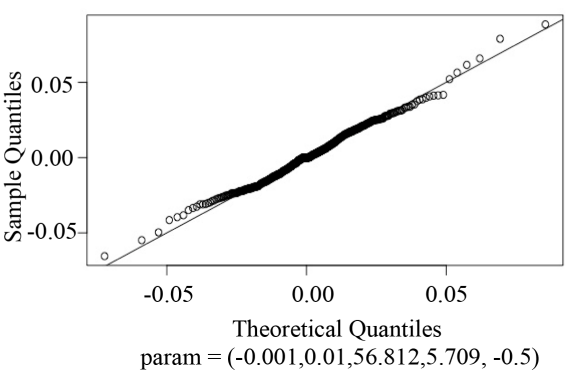

Figure 1. Normal, empirical and NIG data series distribution at pre-crisis scenario. Distribution graphics show normal distribution (Red), empirical data distribution (Blue) and NIG simulated distribution (Green), for interpretation purposes. Graphics were generated in R software.

\section{Conclusion}

Through this analysis, it can be observed that financial returns are reasonably adjusted by the NIG distribution. Particularly, we can use this fact on derivatives valuation which does not have a closed formula of valuation, and it is necessary to employ numerical techniques. The behavior of underlying assets is then a central 


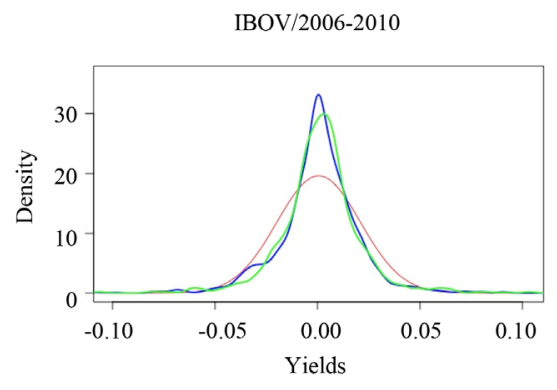

NIFTY50/2006-2010

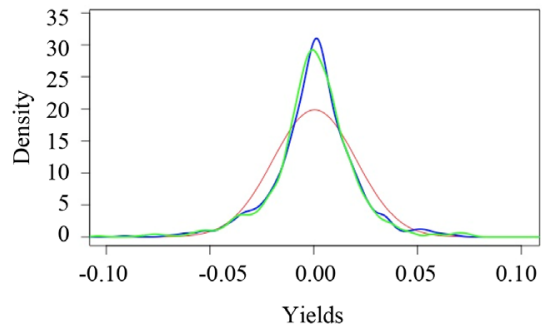

RTSI/2006-2010

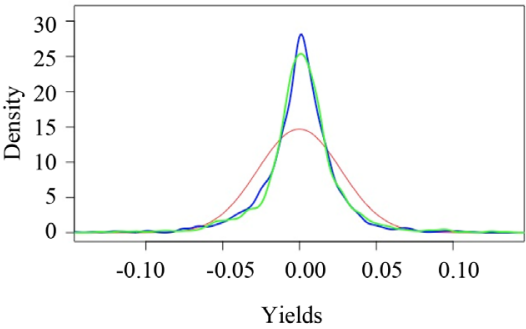

SHCOMP/2006-2010

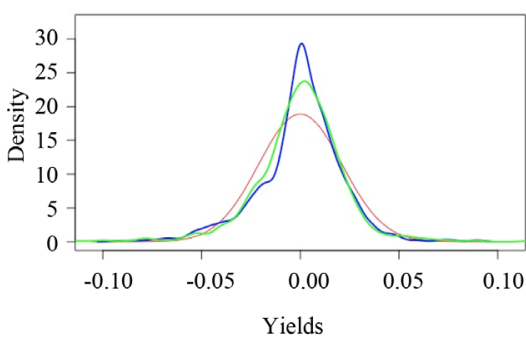

Q-Q Plot of IBOV/2006-2010

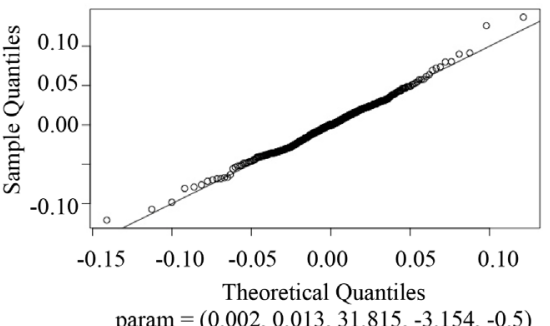

Q-Q Plot of NIFTY50/2006-2010

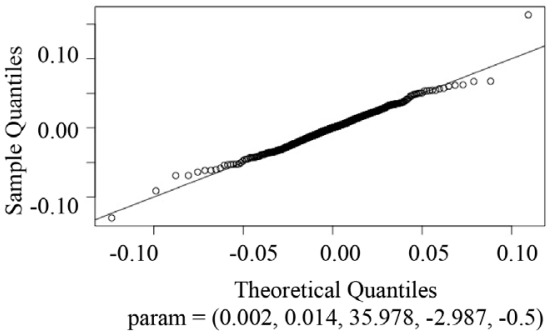

Q-Q Plot of RTSI/2006-2010

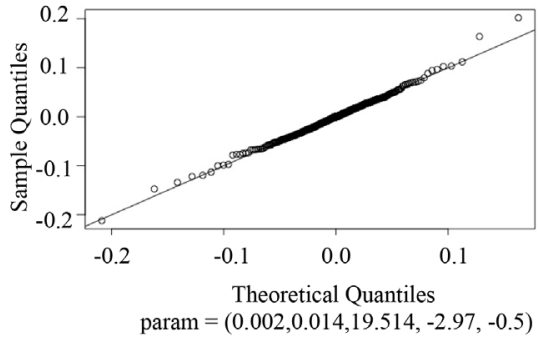

Q-Q Plot of SHCOMP/2006-2010

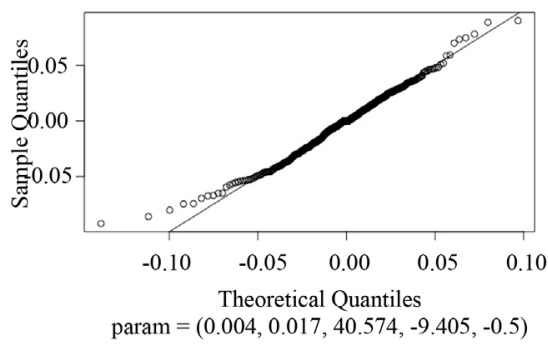

Figure 2. Normal, empirical and NIG data series distribution at crisis scenario. Distribution graphics show normal distribution (Red), empirical data distribution (Blue) and NIG simulated distribution (Green), for interpretation purposes. Graphics were generated in $\mathrm{R}$ software.

issue of this topic. To address this problem, we propose to use the NIG as a distribution that has proven to be an adequate method to fit distribution of stock returns. We have demonstrated to fit the returns of indexes of the BRIC economies as well. Under this basis the indexes return from these economies were analyzed in three periods divided according to different states of nature. Those are pre-crisis, crisis and post-crisis scenarios. These results provide evidence that 
IBOV/2010-2015

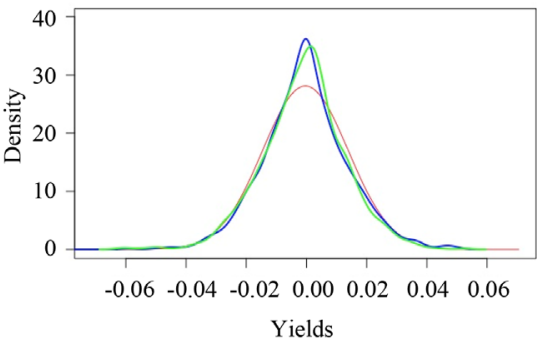

NIFTY50/2010-2015

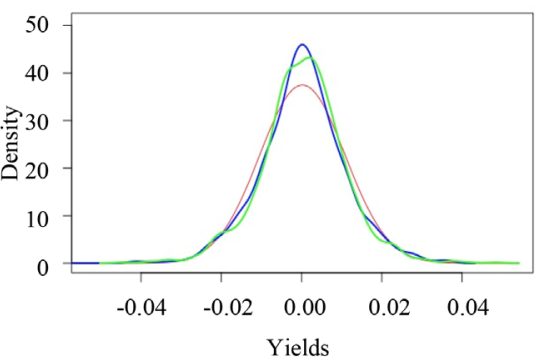

RTSI/2010-2015

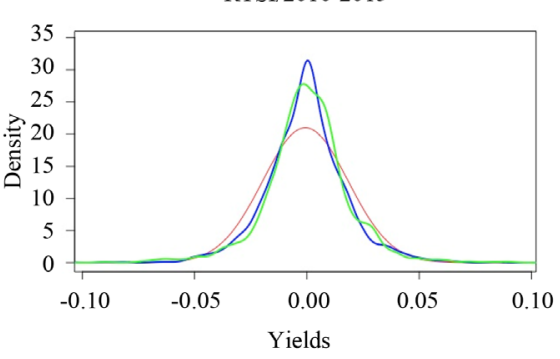

SHCOMP/2010-2015

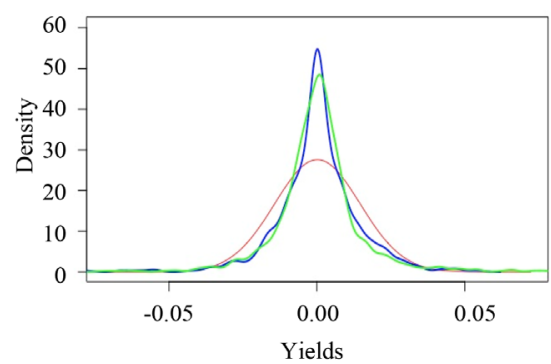

Q-Q Plot of IBOV/2010-2015

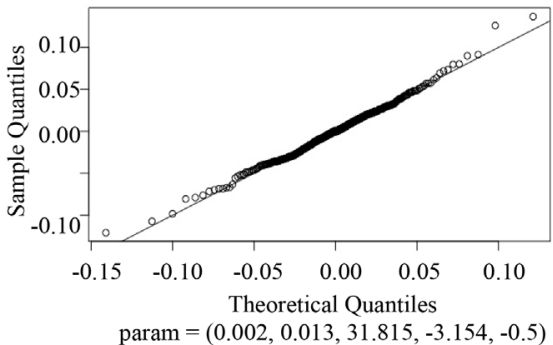

Q-Q Plot of NIFTY50/2010-2015

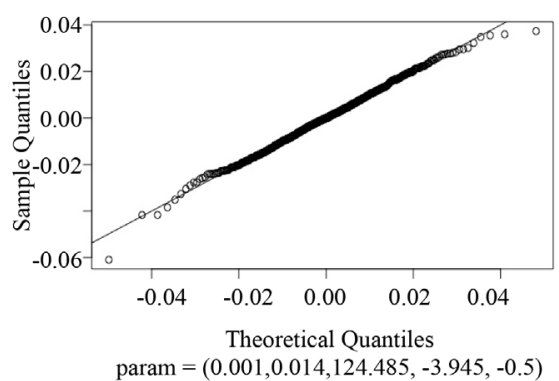

Q-Q Plot of RTSI/2010-2015

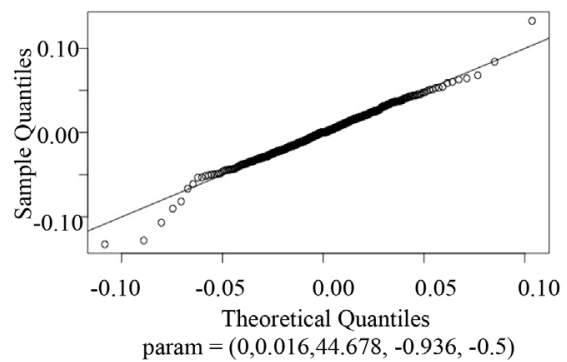

Q-Q Plot of SHCOMP/2010-2015

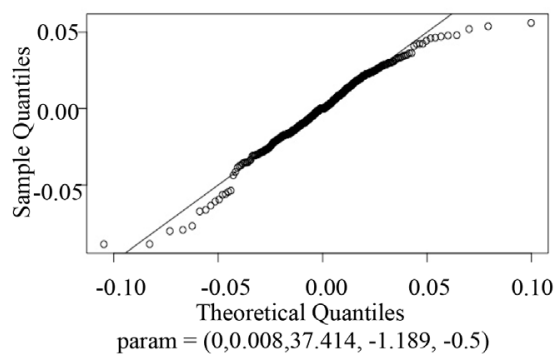

Figure 3. Normal, empirical and NIG data series distribution at post-crisis scenario. Distribution graphics show normal distribution (Red), empirical data distribution (Blue) and NIG simulated distribution (Green), for interpretation purposes. Graphics were generated in $\mathrm{R}$ software.

NIG should be used, or at least tested to model indexes returns in different scenarios, because distribution fits better to empirical data series, even at extreme periods like 2008 financial downturn, which deeply affected all of the world 
Table 4. Likelihood test ( $p$-value).

\begin{tabular}{cccc}
\hline Time Period & Index & Kolmogorov-Smirnov & Anderson-Darling \\
\hline \multirow{2}{*}{$2002-2005$} & IBOV & 0.8758 & 0.7774 \\
& NIFTY50 & 0.2142 & 0.3006 \\
& SHCOMP & 0.0465 & 0.1121 \\
$2006-2010$ & RTSI & 0.0863 & 0.2552 \\
& IBOV & 0.3400 & 0.5358 \\
& NIFTY50 & 0.6029 & 0.8094 \\
& SHCOMP & 0.0890 & 0.3554 \\
$2011-2015$ & RTSI & 0.4937 & 0.3279 \\
& IBOV & 0.2711 & 0.5014 \\
& NIFTY50 & 0.7713 & 0.7679 \\
& SHCOMP & 0.1961 & 0.2523 \\
& RTSI & 0.0681 & 0.1423 \\
\hline
\end{tabular}

Both tests assume a significance level of 0.05 , that means that if $p$-value $\geq 0.05$ null hypothesis is accepted otherwise is rejected and the alternative hypothesis is confirmed. Own elaboration, data processed in R Software.

economies. NIG distribution could be used as outstanding tool for derivatives valuation that uses indexes returns as underlying through Monte Carlo simulations and its variants.

\section{References}

[1] Bachélier, L. (1900) Théorie de la speculation. [The Theory of Speculation.] Annales scientifiques de l'École Normale Supérieure, 3, 21-86. https://doi.org/10.24033/asens.476

[2] Bank for International Settlements (2017) Statistical Release: OTC Derivatives Statistics at End June 2017. BIS Semiannual OTC Derivatives Statistics. https://www.bis.org/publ/otc_hy1711.pdf

[3] Eberlein, E. and Keller, U. (1995) Hyperbolic Distributions in Finance. Bernoulli, 1, 281-299. https://doi.org/10.2307/3318481

[4] Eberlein, E. and Prause, K. (2002) The Generalized Hyperbolic Model: Financial Derivatives and Risk Measures. In: Geman, H., Madan, D., Pliska, S.R. and Vorst, T., Eds., Mathematical Finance-Bachelier Congress 2000, Springer, Berlin, Heidelberg, 245-267. https://doi.org/10.1007/978-3-662-12429-1_12

[5] Cont, R. (2001) Empirical Properties of Asset Returns: Stylized Facts and Statistical Issues. Quantitative Finance, 1, 223-236. https://doi.org/10.1080/713665670

[6] Barndorff-Nielsen, O.E. (1997) Normal Inverse Gaussian Distributions and Stochastic Volatility Modelling. Scandinavian Journal of Statistics, 24, 1-13. https://doi.org/10.1111/1467-9469.t01-1-00045

[7] Barndorff-Nielsen, O.E. and Shephard, N. (2001) Modelling by Lévy Processes for Financial Econometrics. In: Ole, E., Barndorff-Nielsen, T.M. and Sidney, I.R., Eds., Lévy Processes: Theory and Applications, Birkhäuser, Boston, 283-318. https://doi.org/10.1007/978-1-4612-0197-7_13 
[8] Barndorff-Nielsen, O.E. (1977) Exponentially Decreasing Distributions for the Logarithm of Particle Size. Proceedings of the Royal Society A: Mathematical, Physical and Engineering Sciences, 353, 401-419. https://doi.org/10.1098/rspa.1977.0041

[9] Barndorff-Nielsen, O. E. (1995), Normal Inverse Gaussian processes and the Modelling of Stock Returns. Research Report 300, Department of Theoretical Statistics, Institute of Mathematics, University of Aarhus, Aarhus.

[10] Rydberg, T.H. (1999) Generalized Hyperbolic Diffusion Processes with Applications in Finance. Mathematical Finance, 9, 183-201. https://doi.org/10.1111/1467-9965.00067

[11] Trejo, B.R., Núñez, J.A. and Lorenzo, A. (2006) Distribución de los rendimientos del mercado mexicano accionario. Estudios Económicos, 21, 85-118.

[12] Mota, M. and Mata, L. (2016) Caracterización paramétrica de los rendimientos de los precios del petróleo 2010-2015. Panorama Económico, 11, 63-74.

[13] Shen, H., et al. (2017) Heavy-Tailed Distribution and Risk Management of Gold Returns. International Journal of Academic Research in Economics and Management Sciences, 3, 15-24.

[14] Shen, H., Meng, X. and Meng, X. (2017) How to Manage the Risk in the Precious Metals Market? The Case of Gold. https://doi.org/10.2139/ssrn.3016829

[15] Neely, C.J. (2004) The Federal Reserve Responds to Crises: September 11th Was Not the First. ICPSR Data Holdings.

[16] Clark, J. (2016) Emerging Market Capital Flows and U.S. Monetary Policy. Board of Governors of the Federal Reserve System. International Finance Discussion Paper Note.

[17] Shapiro, S.S. and Francia, R.S. (1972) An Approximate Analysis of Variance Test for Normality. Journal of the American Statistical Association, 67, 215-216. https://doi.org/10.1080/01621459.1972.10481232

[18] Anderson, T.W. and Darling, D.A. (1954) A Test of Goodness of Fit. Journal of the American Statistical Association, 49, 765-769.

https://doi.org/10.1080/01621459.1954.10501232 